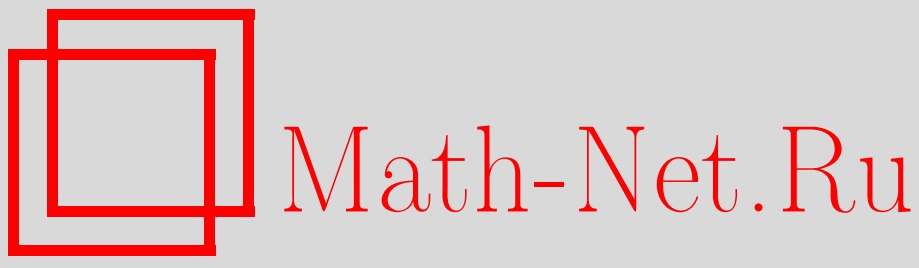

В. Л. Хацкевич, О некоторых нелинейных характеристиках центра группирования случайных величин, Изв. вузов. Матем., 2020, номер 8, 50-58

DOI: https://doi.org/10.26907/0021-3446-2020-8-50-58

Использование Общероссийского математического портала Math-Net.Ru подразумевает, что вы прочитали и согласны с пользовательским соглашением

http://www. mathnet.ru/rus/agreement

Параметры загрузки:

IP : 3.85 .7 .115

26 апреля 2023 г., 08:10:52 
Известия вузов. Математика

2020 , № 8 , c. $50-58$ https://kpfu.ru/science/nauchnye-izdaniya/ivrm

e-mail: izvuz.matem@kpfu.ru

\title{
В.Л. ХАЦКЕВИЧ
}

\section{О НЕКОТОРЫХ НЕЛИНЕЙНЫХ ХАРАКТЕРИСТИКАХ ЦЕНТРА ГРУППИРОВАНИЯ СЛУЧАЙНЫХ ВЕЛИЧИН}

\begin{abstract}
Аннотация. В работе изучены различные аспекты теории нелинейных средних случайных величин, в частности, средних геометрических, средних гармонических и средних степенных.
\end{abstract}

Ключевые слова: нелинейные средние случайных величин, алгебраические и экстремальные свойства, неравенства.

УДК: 519.244

DOI: $10.26907 / 0021-3446-2020-8-50-58$

\section{ВВЕДЕНИЕ}

Наиболее известными характеристиками центра группирования случайных величин являются их математическое ожидание и медиана, однако иногда полезны и другие характеристики. Ниже исследуются некоторые свойства средней геометрической, средней гармонической, а также средней степенной случайных величин. Рассматриваемые случайные величины предполагаются положительными и имеющими конечные математические ожидания.

В работе вводится общее определение нелинейной средней случайной величины, включающее в себя понятие средней геометрической, средней гармонической и средней степенной. Рассматривается задача точечного оценивания нелинейных средних. Демонстрируются экстремальные свойства нелинейных средних. Приводятся алгебраические свойства, аналогичные свойствам математического ожидания. Рассматриваются неравенства между средними. Частично приводимые ниже свойства известны для дискретных аналогов, другие для них не характерны либо содержат отличия. Сравнение с известными результатами приводится по ходу изложения.

Пусть $(\Omega, A, P)$ - некоторое вероятностное пространство (см., например, [1], гл. II, §1). Здесь $\Omega$ - пространство элементарных событий, $A-\sigma$-алгебра подмножеств из $\Omega, P-$ вероятностная мера. Пусть $R$ - множество вещественных чисел. Рассмотрим случайную величину $X: \Omega \rightarrow R$. Через $E X$ будем обозначать ее математическое ожидание. Рассмотрим борелевскую функцию $f(x)$, определенную на множестве действительных чисел $R$. Функцией $Y=f(X)$ случайной величины $X$ называют случайную величину $Y$, полученную суперпозицией действительной функции $X=X(\omega)$, заданной на множестве $\Omega$, и функции $f(x)$. То есть $Y=f(X(\omega))=Y(\omega)$. При этом математическое ожидание $E f(X)$

Поступила в редакцию 10.09.2019, после доработки 10.09.2019. Принята к публикации 18.12.2019. 
определяется интегралом Стилтьеса-Лебега $E f(X)=\int_{-\infty}^{\infty} f(x) d F(x)$, где $F(x)-$ функция распределения случайной величины $X$.

Ниже будем использовать неравенства между случайными величинами $X$ и $Y$, заданными на одном вероятностном пространстве $(\Omega, A, P)$, понимая $X \geq Y$ как $X(\omega) \geq Y(\omega)$ для всех элементарных исходов $\omega \in \Omega$.

Среднее геометрическое значение $G(X)$ случайной величины $X(X>0)$ задается формулой (см., например, [2], гл. 5, п. 5.6.2)

$$
G(X)=e^{E(\ln X)},
$$

среднее гармоническое $H(X)(X>0)$ - формулой

$$
H(X)=1 /(E(1 / X)) .
$$

Отметим, что среднее геометрическое значение используют при изучении процессов, рост которых пропорционален уже достигнутому уровню (рост численности населения, валового продукта и т. п.), а также при расчетах индексов цен.

Среднее гармоническое $H(X)$ используют в экономике в индексных расчетах.

Наряду со средними геометрическими и средними гармоническими будем также рассматривать средние степенные.

Как известно, средняя степенная $p$-го порядка $(p>0),(X>0)$ определяется формулой

$$
E_{p}(X)=\left(E\left(X^{p}\right)\right)^{1 / p} .
$$

Средняя степенная $p$-го порядка - это есть $p$-й начальный момент случайной величины $X$, возведенный в степень $1 / p$, т. е. норма в пространстве $L_{p}(\Omega, A, P)$ (для случая $X>0$ ). Средняя степенная в случае $p=1$ превращается в математическое ожидание.

В качестве примера рассмотрим дискретную случайную величину $X$, имеющую равномерное распределение. Пусть она принимает различные положительные значения $x_{1}<x_{2}<$ $\ldots<x_{n}$ с вероятностями $p_{i}=P\left(X=x_{i}\right)=1 / n, i=1,2, \ldots, n$. Тогда

$$
\begin{gathered}
G(X)=\hat{G}\left(x_{1}, x_{2}, \ldots, x_{n}\right)=\sqrt[n]{x_{1} x_{2} \ldots x_{n}}, \\
H(X)=\hat{H}\left(x_{1}, x_{2}, \ldots, x_{n}\right)=\left(\frac{1}{n} \sum_{i=1}^{n} \frac{1}{x_{i}}\right)^{-1}, \\
E_{p}(X)=\hat{E}_{p}\left(x_{1}, x_{2}, \ldots, x_{n}\right)=\left(\frac{1}{n} \sum_{i=1}^{n} x_{i}^{p}\right)^{1 / p},
\end{gathered}
$$

т. е. соответственно средняя геометрическая, средняя гармоническая и средняя степенная совокупности чисел $x_{1}, \ldots, x_{n}$. Знак «колпачок» над средней здесь и ниже обозначает дискретную (эмпирическую) среднюю.

Если в этом примере $P\left(X=x_{i}\right)=p_{i}$, то получим соответственно взвешенную среднюю геометрическую, среднюю гармоническую и среднюю степенную с весами $p_{i}$.

Замечание 1. Для средней геометрической (1) по определению справедливо следующее характерное свойство. Для любого $\alpha>0$ имеет место равенство $G\left(X^{\alpha}\right)=(G(X))^{\alpha}$.

Аналогичное свойство справедливо и для дискретной средней геометрической (4). 


\section{1. АСИМПТОТИКИ И ЭКСТРЕМАЛЬНЫЕ СВОЙСТВА}

Обозначим через $R_{+}$вещественную полуось $(0, \infty)$. Вышеприведенные формулы $(1)-(3)$ для нелинейных средних можно объединить в одну.

Пусть $\phi: R_{+} \rightarrow R-$ вещественная строго монотонная непрерывная функция. В силу предположений существует однозначная обратная функция $\phi^{-1}$. Определим нелинейное среднее $\tilde{E}_{\phi}(X)$ случайной величины $X>0$ выражением

$$
\tilde{E}_{\phi}(X)=\phi^{-1}(E \phi(X))
$$

предполагая, что существует конечное математическое ожидание $E \phi(X)$.

Фигурирующую в (7) функцию $\phi$ будем называть определяющей функцией. В случае $\phi(x)=\ln x, \phi^{-1}(y)=e^{y}$ имеем $\tilde{E}_{\phi}(X)=G(X)$ - среднюю геометрическую. Соответствующую определяющую функцию обозначим $\phi_{G}$. В случае $\phi(x)=x^{p}, \phi^{-1}(y)=y^{1 / p}$ имеем $\tilde{E}_{\phi}(X)=E_{p}(X)-$ среднюю степенную. Соответствующую определяющую функцию обозначим $\phi_{p}$. В случае $\phi(x)=1 / x, \phi^{-1}(y)=y^{-1}$ имеем $\tilde{E}_{\phi}(X)=H(X)-$ среднюю гармоническую. Соответствующую определяющую функцию обозначим $\phi_{H}$.

Пусть $X_{1}, X_{2}, \ldots, X_{n}$ - выборка объема $n$, полученная в результате повторных независимых наблюдений за случайной величиной $X$. По определению все случайные величины $X_{1}, X_{2}, \ldots, X_{n}$ независимы и распределены одинаково, так же как случайная величина $X$.

В качестве оценки средней $\tilde{E}_{\phi}(X)$ рассмотрим

$$
\hat{E}_{\phi}\left(X_{1}, X_{2}, \ldots, X_{n}\right)=\phi^{-1}\left(\frac{1}{n} \sum_{i=1}^{n} \phi\left(X_{i}\right)\right) .
$$

Заметим, что (8) - есть широко известная формула средней общего вида (см., например, [3], гл. III, §3.1; [4], гл. I, §5). Положив в формуле $(8): \phi(x)=\ln x$, получим среднюю геометрическую

$$
\hat{G}\left(X_{1}, X_{2}, \ldots, X_{n}\right)=\sqrt[n]{X_{1} X_{2} \ldots X_{n}}
$$

$\phi(x)=1 / x$, получим среднюю гармоническую

$$
\hat{H}\left(X_{1}, X_{2}, \ldots, X_{n}\right)=\left(\frac{1}{n} \sum_{i=1}^{n} \frac{1}{X_{i}}\right)^{-1},
$$

$\phi(x)=x^{p}$, получим среднюю степенную

$$
\hat{E}_{p}\left(X_{1}, X_{2}, \ldots, X_{n}\right)=\left(\frac{1}{n} \sum_{i=1}^{n} X_{i}^{p}\right)^{1 / p} .
$$

На конкретной реализации $x_{1}, x_{2}, \ldots, x_{n}$ выборки $X_{1}, X_{2}, \ldots, X_{n}$ формулы (9)-(11) совпадают с (4)-(6).

Отметим взаимосвязь между формулами (7) и (8). В формуле (7) аргумент у функции $\phi^{-1}$ из формулы (8) заменен на математическое ожидание $E \phi(X)$.

Аналогом свойства несмещенности для оценки (8) является

Утверждение 1. Пусть непрерывная функиия $\phi(x)$ определена и строго монотонна на $(0, \infty), X$ - положительная случайнал величина. Существует конечное математическое ожсиание $E \phi(X)$ случайной величины $\phi(X)$. Тогда

$$
\phi^{-1}\left(E \frac{1}{n} \sum_{i=1}^{n} \phi\left(X_{i}\right)\right)=\phi^{-1}(E \phi(X)) .
$$


Действительно, в силу непрерывности функции $\phi$ случайные величины $\phi\left(X_{i}\right)$ независимы, а в силу строгой монотонности $\phi$ они одинаково распределены (так же, как $\phi(X)$ ). Поэтому $E \phi\left(X_{i}\right)=E \phi(X)(i=1,2, \ldots, n)$. Тогда $E\left(\frac{1}{n} \sum_{i=1}^{n} \phi\left(X_{i}\right)\right)=\frac{1}{n} \sum_{i=1}^{n} E \phi\left(X_{i}\right)=E \phi(X)$.

Отсюда ввиду строгой монотонности функции $\phi^{-1}$ следует высказанное утверждение.

Оценка (8) является состоятельной. Обоснование этого факта опирается на следующее утверждение, доказанное в [5].

Утверждение 2. Пусть функиия $\phi(x)$ непрерывна и строго возрастает (либо убывает) на $(0, \infty)$. Пусть $\xi_{1}, \xi_{2}, \ldots, \xi_{n}$ - семейство одинаково распределенных, попарно независимых случайных величин. Существует конечное математическое ожидание $E \phi\left(\xi_{1}\right)=b$. Тогда $\phi^{-1}\left(\sum_{i=1}^{n} \frac{1}{n} \phi\left(\xi_{i}\right)\right) \rightarrow \phi^{-1}(b)$ при $n \rightarrow \infty$ с вероятностью 1.

Следствие 1. Оценки (9)-(11) состоятельны.

Утверждение 2 представляет собой вариант так называемых теорем непрерывности (см., например, [6], гл. I, §5).

По поводу эффективности оценки (8) (соответственно оценок (9)-(11)) приведем следующее

Замечание 2. Оценка $\bar{Y}_{n}=\frac{1}{n} \sum_{i=1}^{n} \phi\left(X_{i}\right)$ эффективна в классе всех линейных оценок вида $Y_{n}=\sum_{i=1}^{n} \alpha_{i} \phi\left(X_{i}\right)$ с вещественными коэффициентами $\alpha_{i}$ такими, что $E Y_{n}=E \phi(X)$. А именно,

$$
E\left(\bar{Y}_{n}-E \phi(X)\right)^{2}=\min _{Y_{n}} E\left(Y_{n}-E \phi(X)\right)^{2} .
$$

Доказательство опирается на факт попарной независимости случайных величин $\phi\left(X_{i}\right)$ и равенство $E \phi\left(X_{i}\right)=E \phi(X) \quad(i=1,2, \ldots, n)$. С учетом этого задача сводится к отысканию минимума функции $\sum_{i=1}^{n} \alpha_{i}^{2}$ при условии $\sum_{i=1}^{n} \alpha_{i}=1$. Решение этой задачи дает требуемый результат (ср., например, [7], гл. I, §6).

Рассмотрим экстремальные свойства нелинейных средних характеристик вида (7).

Утверждение 3. Для заданной случайной величины $X>0$ значение $a_{0}=\phi \tilde{E}_{\phi}(X)$ минимизирует среднюю квадратическую ошибку $E(\phi(X)-a)^{2}$ по $a \in R$.

Действительно, по определению $a_{0}=\phi\left(\tilde{E}_{\phi}(X)\right)=E \phi(X)$. Тогда согласно экстремальному свойству математических ожиданий (см., например, [1], гл. I, §4), примененному к случайной величине $\phi(X)$, получим

$$
\min _{a} E(\phi(X)-a)^{2}=E(\phi(X)-E \phi(X))^{2} .
$$

Что и влечет высказанное утверждение.

Для средней геометрической это означает, что среднее квадратическое отклонение натурального логарифма положительной случайной величины $(\ln X)$ от натурального логарифма средней геометрической $(\ln G(X))$ не больше среднего квадратического отклонения $\ln X$ от любой другой вещественной постоянной. Для средней степенной порядка $p$ это означает, что среднее квадратическое отклонение $p$-й степени случайной величины $X\left(X^{p}\right)$ от 
начального момента $\left(E X^{p}\right)$ не более среднего квадратического отклонения $X^{p}$ от любой вещественной постоянной. Для средней гармонической $H(X)$ это означает, что среднее квадратическое отклонение $X^{-1}$ от $H(X)^{-1}$ не более среднего квадратического отклонения $X^{-1}$ от любой другой вещественной постоянной.

Для дискретных аналогов (4)-(6) соответствующие свойства отмечены, например, в ([4], гл. I, §5).

\section{2. НЕКОТОРЫЕ АЛГЕБРАИЧЕСКИЕ СВОЙСТВА НЕЛИНЕЙНЫХ СРЕДНИХ}

Ниже мы перечислим свойства нелинейных характеристик (1)-(3), близкие к свойствам математических ожиданий, и укажем отличия.

Свойство 1. Значение средней (7) от положительной постоянной - есть сама эта постоянная.

Действительно, пусть $C>0-$ некоторая постоянная. Указанное свойство следует из формулы (7) посредством выкладки

$$
\tilde{E}_{\phi}(C)=\phi^{-1}(E \phi(C))=\phi^{-1}(\phi(C))=C .
$$

В частности, $G(C)=C, H(C)=C$ и $E_{p}(C)=C$.

Свойство 2 (однородностъ). $G(X), H(X)$ и $E_{p}(X)$ являются положительно однородными.

Приведем рассуждения для средней гармонической. Пусть $X-$ положительная случайная величина. Для произвольного $k>0$ можем записать

$$
H(k X)=\frac{1}{E(1 / k X)}=\frac{1}{E\left(\frac{1}{k} \cdot \frac{1}{X}\right)}=\frac{1}{\frac{1}{k} E(1 / X)}=k H(X) .
$$

Для средней геометрической это утверждение будет следовать из свойства мультипликативности, для средней степенной - из ее определения.

Свойство 3 (мультипликативностъ). Для произвольных положительных случайных величин $X$ и $Y$, при условии существования математических ожиданий всех фигурирующих случайных величин, имеет место равенство $G(X Y)=G(X) G(Y)$. При дополнительном предположении о независимости случайных величин $X$ и $Y$ справедливы равенства $H(X Y)=H(X) H(Y)$ и $E_{p}(X Y)=E_{p}(X) E_{p}(Y)$.

Действительно, для средней геометрической получим

$$
G(X Y)=e^{E(\ln (X Y))}=e^{E(\ln X+\ln Y)}=e^{E \ln X+E \ln Y}=e^{E \ln X} e^{E \ln Y}=G(X) G(Y) .
$$

В частности, если $Y=C$ - постоянная, то $G(X C)=G(X) G(C)=G(X) C$.

Рассмотрим это свойство для средней гармонической. Пусть случайные величины $X>0$ и $Y>0$ независимы. Тогда

$$
H(X Y)=E\left(\frac{1}{X Y}\right)^{-1}=\left(E\left(\frac{1}{X} \cdot \frac{1}{Y}\right)\right)^{-1}=\left(\left(E \frac{1}{X}\right)\left(E \frac{1}{Y}\right)\right)^{-1}=H(X) \cdot H(Y) .
$$

Здесь мы использовали независимость случайных величин $\frac{1}{X}$ и $\frac{1}{Y}$, вытекающую из независимости $X$ и $Y$.

Аналогичная выкладка справедлива для средней степенной.

Свойство 3 показывает, что среднюю геометрическую удобно использовать в мультипликативных моделях.

Отметим, что из дискретных аналогов свойство 3 верно лишь для средней геометрической (4). 
Замечание 3. Как известно (см., [1], гл. II, §6), для чисел $p, q>1$ таких, что $\frac{1}{p}+\frac{1}{q}=1$, справедливо неравенство Гёльдера. В наших обозначениях оно имеет вид $E(X Y) \leq E_{p}(X) E_{q}(Y)$.

Для средней гармонической это влечет соотношение $H(X Y)=E\left(\frac{1}{X} \frac{1}{Y}\right)^{-1} \geq\left(E_{p}\left(\frac{1}{X}\right)\right)^{-1} \times$ $\times\left(E_{q}\left(\frac{1}{Y}\right)\right)^{-1}=\left(H\left(X^{p}\right)\right)^{1 / p}\left(H\left(Y^{q}\right)\right)^{1 / q}$.

Свойство 4 (монотонное возрастание). Пусть случайные величины $X$ и $Y$ таковы, что $X>Y>0$, и существуют конечные математические ожидания $\phi(X)$ и $\phi(Y)$. Тогда для соответствующих средних (7) выполнено неравенство $\tilde{E}_{\phi}(X)>\tilde{E}_{\phi}(Y)$.

Действительно, предположим сначала, что определяющая функция $\phi$ монотонно возрастает.

Поскольку $X>Y$, то в силу монотонного возрастания функции $\phi$ имеем $\phi(X)>\phi(Y)$. И по свойству математических ожиданий $E(\phi(X))>E(\phi(Y))$. Тогда в силу монотонного возрастания $\phi^{-1}$ получим требуемый результат.

Аналогично рассматривается случай, когда функция $\phi$ монотонно убывает: если $X>Y$, то $\phi(X)<\phi(Y)$. Тогда $E \phi(X)<E \phi(Y)$ и в силу монотонного убывания функции $\phi^{-1}$ получим $\phi^{-1}(E \phi(X))>\phi^{-1}(E \phi(Y))$, что и требовалось.

Следствие 2. Величины $G(X), H(X)$ и $E_{p}(X)$ монотонно возрастают в классе положительных случайных величин.

Отметим, что указанные выше свойства 1-4 аналогичны свойствам математических ожиданий.

Следствие 3. Пусть $X$ и $Y$ - положительные случайные величины. Тогда

$$
2 \tilde{E}_{\phi}(\min \{X, Y\}) \leq \tilde{E}_{\phi}(X+Y) \leq 2 \tilde{E}_{\phi}(\max \{X, Y\})
$$

в предположении, что существуют все необходимые конечные математические ожидания.

Следствие 4 (аналог неравенства Коши). Пусть $X$ и $Y$ - положительные случайные величины. Для средней геометрической, а также для средней гармонической и средней степенной при дополнительном предположении о независимости $X$ и $Y$ верно неравенство $\tilde{E}_{\phi}(X+Y) \geq 2 \tilde{E}_{\phi}(\sqrt{X}) \tilde{E}_{\phi}(\sqrt{Y})$ для соответствующих определяющих функций $\phi_{G}, \phi_{H}, \phi_{p}$.

При этом предполагается существование всех необходимых математических ожиданий.

Рассмотрим случай средней геометрической. Поскольку $X+Y \geq 2 \sqrt{X Y}$, то согласно свойству $4 G(X+Y) \geq G(2 \sqrt{X Y})$. При этом

$$
G(2 \sqrt{X Y})=e^{E \ln (2 \sqrt{X Y})}=e^{E(\ln 2+\ln \sqrt{X}+\ln \sqrt{Y})}=e^{E \ln 2} e^{E \ln \sqrt{X}} e^{E \ln \sqrt{Y}}=2 G(\sqrt{X}) G(\sqrt{Y}),
$$

что и влечет высказанное утверждение в случае средней геометрической.

Предположим дополнительно, что случайные величины $X$ и $Y$ независимы. Продемонстрируем рассуждения для средней степенной. Поскольку $X+Y \geq 2 \sqrt{X Y}$, то с учетом свойства $4 E_{p}(X+Y) \geq E_{p}(2 \sqrt{X} \sqrt{Y})=2 E_{p}(\sqrt{X}) E_{p}(\sqrt{Y})$. Последнее равенство справедливо в силу свойства 3 . Аналогично для средней гармонической $H(X+Y) \geq 2 H(\sqrt{X}) H(\sqrt{Y})$.

Отметим, что в силу замечания 1 и следствия 4 неравенство для средней геометрической можно записать в форме $G(X+Y) \geq 2 \sqrt{G(X)} \sqrt{G(Y)}$.

Как известно ([1], гл. II, §6), для средней степенной при $p>1$ справедливо неравенство Минковского $E_{p}(X+Y) \leq E_{p}(X)+E_{p}(Y)$. При дополнительном предположении о независимости случайных величин $X$ и $Y$ следствие 4 для средних степенных дает оценку $E_{p}(X+Y)$ снизу. 


\section{3. НЕРАВЕНСТВА МЕЖДУ СРЕДНИМИ}

Как известно (см., например, [3], гл. II, §2.5; [4], гл. I, §7), справедливо знаменитое неравенство $\hat{H}(X) \leq \hat{G}(X) \leq \hat{E}(X)$ между эмпирическими средними (4)-(6), где $\hat{E}(X)-$ средняя степенная при $p=1$, т. е. математическое ожидание. Более того, для любого $p>0$ справедливо неравенство $\hat{H}(X) \leq \hat{G}(X) \leq \hat{E}_{p}(X)$. Покажем, какой вид примут эти неравенства для теоретических нелинейных средних (1)-(3), и укажем отличия.

Сначала приведем утверждения общего характера о соотношении нелинейных средних (7) и математического ожидания $E X$. Заметим, что определяющие функции $\phi_{G}, \phi_{H}$ и $\phi_{p}$, кроме свойств строгой монотонности, обладают определенными свойствами выпуклости. В связи с этим сформулируем важное для дальнейшего неравенство Иенсена (см., например, [1], гл. II, §6): если $\phi(x)$ - выпуклая вверх (вогнутая) борелевская функция, то для любой случайной величины $X$, для которой существует математическое ожидание, выполнено неравенство $E(\phi(X)) \leq \phi(E X)$; если $g(x)$ - выпуклая вниз борелевская функция, то имеет место противоположное неравенство $E(g(X)) \geq g(E X)$.

Утверждение 4. Пусть определяющая функиия ф в (7) непрерывна, выпукла вверх и строго монотонно возрастает на $(0,+\infty)$ либо выпукла вниз и строго монотонно убывает на $(0,+\infty)$. Тогда для любой положительной случайной величины $X$ имеет место неравенство $\tilde{E}_{\phi}(X) \leq E X$. Пусть определяющал функция выпукла вниз и строго монотонно возрастает на $(0,+\infty)$. Тогда $\tilde{E}_{\phi}(X) \geq E X$. При этом предполагается существование всех необходимых математических ожиданий.

Проведем рассуждения для одного из вариантов. Пусть, например, функция $\phi$ выпукла вверх и строго монотонно возрастает на $(0,+\infty)$. Пусть $X-$ произвольная положительная случайная величина и существуют математические ожидания $E X$ и $E(\phi(X))$. По предположению $E X>0$, а по неравенству Иенсена $E(\phi(X)) \leq \phi(E X)$. Тогда в силу строго монотонного убывания функции $\phi^{-1}$ получим $\tilde{E}_{\phi}(X) \leq E X$.

Следствие 5. В предположении о существовании всех необходимых математических ожиданий для положительной случайной величины $X$ имеем $G(X) \leq E X, E_{p}(X) \leq E X$ при $p<1, H(X) \leq E X$ и $E_{p}(X) \geq E X$ при $p>1$.

Теорема. Пусть $X-$ положительная случайная величина. B предположении о существовании всех необходимых математических ожиданий для нелинейных средних справедливо соотношение

$$
H(X) \leq G(X) \leq E(X) .
$$

Отметим, что правая часть неравенства (13) известна (см., например, [2], гл. 5, п. 5.6.2), где приведена формулировка. Мы ее привели в следствии 5.

Покажем, что справедливо неравенство

$$
H(X) \leq G(X)
$$

Заметим, что по определению

$$
H(X)=\frac{1}{E(1 / X)}=e^{\ln (E(1 / X))^{-1}}=e^{-\ln (E(1 / X))} .
$$

Положим $Y=1 / X$. Так как $\ln y$ выпуклая вверх по $y>0$ функция, то по неравенству Иенсена имеем $\ln (E Y) \geq E(\ln Y)$, т. е.

$$
\ln (E(1 / X)) \geq E \ln (1 / X)=-E \ln (X) .
$$


Следовательно, $-\ln (E(1 / X)) \leq E \ln (X)$. Тогда согласно (15) и в силу монотонного возрастания экспоненты

$$
H(X)=e^{-\ln (E(1 / X))} \leq e^{E \ln (X)}=G(X) .
$$

С учетом следствия 5 теорема влечет

Следствие 6. В условиях теоремы и при $p>1$ имеет место соотношение

$$
H(X) \leq G(X) \leq E(X) \leq E_{p}(X)
$$

Отметим, что неравенство $E(X) \leq E_{p}(X)$ при $p>1$ вытекает также из неравенства моментов (см., например, [1], гл. II, §6).

Отметим, что в отличие от эмпирических средних в случае $0<p<1$, справедливо

Замечание 4. При $0<p<1$ для положительной случайной величины $X$, имеющей конечные математические ожидания $E\left(\phi_{G}(X)\right)$ и $E\left(\phi_{p}(X)\right)$, справедливо неравенство

$$
G(X) \geq E_{p}(X)
$$

Действительно, рассмотрим представление

$$
E_{p}(X)=\left(E X^{p}\right)^{1 / p}=e^{\ln \left(E X^{p}\right)^{1 / p}}=e^{\frac{1}{p} \ln \left(E X^{p}\right)} .
$$

Далее, используем неравенство Иенсена, беря в качестве $\phi(x)$ функцию $x^{p}$ для $x>0$ при $0<p<1$. Она выпукла кверху. Поэтому $E\left(X^{p}\right) \leq(E X)^{p}$. Тогда

$$
\ln \left(E X^{p}\right) \leq \ln (E X)^{p}=p \ln (E X) .
$$

То есть $\frac{1}{p} \ln \left(E X^{p}\right) \leq \ln (E X)$. Так как экспонента монотонно возрастает, то $E_{p}(X) \leq e^{\ln (E X)}=$ $G(X)$, что и требовалось.

Отметим еще раз, что при $p \geq 1$ согласно (16) наоборот, $E_{p}(X) \geq G(X)$.

Предельную взаимосвязь между средними $E_{p}(X)$ и $G(X)$ описывает

Утверждение 5. Для произвольной положительной случайной величины $X$, для которой существуют конечные математические ожидания $E(X)$ и $E\left(\phi_{G}(X)\right)$, справедливо соотношение $\lim _{p \rightarrow 0} E_{p}(X)=G(X)$.

Приведем схему доказательства. Заметим, что в силу неравенства моментов из существования $E(X)$ вытекает существование $E\left(X^{p}\right)$ при $p<1$. Рассмотрим $\ln E_{p}(X)=\frac{1}{p} \ln E\left(X^{p}\right)$. Вычисляя предел от правой части при $p \rightarrow 0$, с помощью правила Лопиталя получим

$$
\lim _{p \rightarrow 0} \frac{\ln \left(E\left(X^{p}\right)\right)}{p}=E \ln X .
$$

Тогда

$$
\lim _{p \rightarrow 0} E_{p}(X)=\lim _{p \rightarrow 0} e^{\ln E_{p}(X)}=e^{\lim _{p \rightarrow 0} \ln E_{p}(X)}=e^{E \ln X}=G(X) .
$$

Этот результат аналогичен известному для дискретных средних (6) и (4) (см., например, [4], гл. I, §2). 


\section{4. ЗАКЛЮЧЕНИЕ}

В нашей работе выделен новый класс нелинейных средних случайных величин вида (7), включающих в себя среднюю геометрическую, среднюю гармоническую и среднюю степенную, и изучены его свойства. Эти результаты могут быть полезны, в частности, при эконометрическом анализе. В качестве возможных направлений развития установленных результатов укажем на перспективное исследование взаимосвязи с нелинейным законом больших чисел (см., [5] и [8], гл. $5, \S 5.5)$, а также изучение свойств ковариаций и дисперсий, порожденных нелинейными средними.

\section{ЛитеРАТУРА}

[1] Ширяев А.Н. Вероятность (Наука, М., 1980).

[2] Айвазян С.А., Енюков И.Е., Мешалкин Л.Д. Прикладная статистика. Основъ моделирования и первичная обработка данных (Финансы и статистика, М., 1983).

[3] Харди Г.Г., Пойа Д., Литтлвуд Д.И. Неравенства (МЦМНО, М., 2008).

[4] Джини К. Средние величинъ (Статистика, М., 1970).

[5] Хацкевич В.Л. О законе больших чисел для нелинейных средних случайнъх величин, Изв. вузов. Матем., №8, 79-87 (2019).

[6] Боровков А.А. Математическал статистика (URSS, M., 2018).

[7] Розанов Ю.А. Теория вероятностей, случайнъе процессы и математическая статистика (Наука, М., 1989).

[8] Орлов А.И. Прикладная статистика (Изд-во «Экзамен», М., 2006).

Владимир Львович Хацкевич

Военный учебно-научный центр Военно-воздушных сил

"Военно-воздушная академия им. Н.Е. Жуковского и Ю.А. Гагарина",

ул. Старых Большевиков, д. 54 а, г. Воронеж, 394064, Россия,

e-mail : vlkhats@mail.ru

\section{V.L. Khatskevich}

\section{On some nonlinear characteristics of the center of grouping of random variables}

Abstract. The paper devoted to diffferent aspects of the theory of mean random variables, i.e., geometric mean, harmonic mean and average power.

Keywords: mean random variables, geometric mean, harmonic mean, average power.

Vladimir L'vovich Khatskevich

Military educational scientific center Air force

"Air force academy named after N.E. Zhukovsky and Yu.A. Gagarin",

54 a old Bolsheviks str., Voronezh, 394064 Russia,

e-mail:vlkhats@mail.ru 